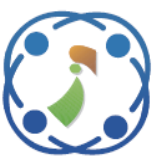

\title{
System Performance Enhancement with Energy Efficiency Based Sleep Control for 5G Heterogeneous Cellular Networks
}

\author{
Arif Dataesatu $^{1} \quad$ Kosuke Sanada $^{1} \quad$ Hiroyuki Hatano $^{1} \quad$ Kazuo Mori $^{1 *}$ \\ Pisit Boonsrimuang ${ }^{2}$ \\ ${ }^{I}$ Department of Electrical and Electronic Engineering, Graduate School of Engineering, \\ Mie University, Mie, 514-8507 Japan \\ ${ }^{2}$ Department of Telecommunication Engineering, School of Engineering, KMITL, Bangkok, 10520 Thailand \\ * Corresponding author's Email: kmori@elec.mie-u.ac.jp
}

\begin{abstract}
This paper presents an improved sleep control algorithm for small base stations (SBSs) in 5G New Radio (NR) heterogeneous cellular networks (HetNets). HetNets consist of various base station tiers, including macro base stations (MBSs) and small base stations (SBSs), and have been suggested as a promising solution to enhance wireless coverage and network capacity, employing many SBSs into the MBS coverage. However, power consumption increases significantly as a result of an increase in the number of the SBSs. To solve this problem, the SBS sleep control has been proposed to reduce power consumption for the SBSs and improves energy efficiency, whereas it deteriorates system throughput compared with no sleep control system, consequently degrading the quality of service (QoS) performance at user equipments (UEs). This paper proposes an enhanced algorithm for SBS sleep control based on energy efficiency as a decision criterion for SBS operating state. From the evaluation results through computer simulation, the proposed scheme can provide improved performance for both energy efficiency and system throughput simultaneously, that is it can improve energy efficiency while maintaining almost the same system throughput as the no sleep control system. Concretely, the proposed scheme has the $14.89 \%$ improvement in energy efficiency while providing almost the same system throughput of over $99 \%$, compared with no sleep control system.
\end{abstract}

Keywords: Sleep control, HetNets, 5G NR, System throughput, Power consumption, Energy efficiency.

\section{Introduction}

The three majors of use cases for the fifth generation new radio (5G NR) mobile networks include enhanced mobile broadband (eMBB), massive machine type communications (mMTC), and ultra-reliable and low latency communications (URLLC) [1]. There are different key performance indicators for different use cases. The userexperienced data rate is essential for eMBB, whereas mMTC is mainly concerned with the density of connections and the main parameters for URLLC are latency and reliability.

Considering that $5 \mathrm{G}$ NR mobile networks need to achieve all three requirements and should be capable of interconnecting with all existing and emerging technologies, one of the most promising solutions for overcoming these challenges is attempting densification of base station (BS) deployment through heterogeneous cellular networks (HetNets), which include different tiers of BSs, macro base stations (MBSs) and small base stations (SBSs), as shown in Fig. 1 [2-4]. MBSs provide low-frequency carrier and high-transmission power to achieve wider coverage with a range of over one kilometer. In contrast, SBSs provide high-frequency carrier and low-power radio access for usually ten to a few hundred meters of coverage. They are classified as femtocells, picocells, and microcells with the smallest being femtocells and the largest being microcells [3]. The SBS tier generally overlays on the MBS tier in the HetNets. It is necessary to balance service quality and cost efficiency while planning and deploying HetNets. 


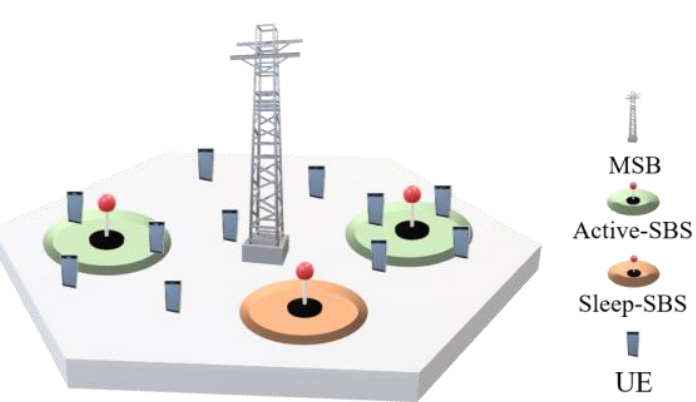

Figure. 1 The example of cell deployment for 5G NR heterogeneous cellular networks

Low cost covers power consumption cost, as well as network planning and operating charges. The implementation of SBSs can achieve both better user quality of service (QoS) and lower operating costs [4].

However, dense and random implementation of SBSs inside the MBS coverage leads to various negative impacts, including a rise in power consumption due to the inclusion of many SBSs [5]. Therefore, various techniques for reducing power consumption in cellular networks have been proposed. [6-7]. One of the most attractive techniques for reducing power consumption and increasing energy efficiency is to integrate sleep control into SBSs. The existing schemes for SBS sleep control have been proposed, for example: random and repulsive schemes [8], distance-sensitive repulsive sleeping strategy [9], traffic based sleep control scheme [10], cell throughput based sleep control scheme [11], and adaptive SBS sleep control scheme [12]. Although these schemes can reduce power consumption and improve energy efficiency, they could degrade system throughput when compared with the no sleep control system.

This paper proposes an enhanced SBS sleep control scheme for downlink communications in the $5 \mathrm{G}$ HetNets in order to provide better performance for both energy efficiency and system throughput simultaneously, concretely improve energy efficiency while maintaining almost the same system throughput as the no sleep control system. To achieve these objectives, this paper proposes an enhanced algorithm for the SBS sleep control, which activates more SBSs while providing the improved energy efficiency. However, increasing the number of active SBSs could lead to increased power consumption and then decreased energy efficiency. Based on this perspective, the proposed scheme employs energy efficiency as the decision criteria for SBS sleeping in order to provide the improved energy efficiency.

The remainder of this paper is structured as follows: Section 2 presents related work to the SBS sleep control and then provides detailed description on the conventional cell throughput based sleep control and adaptive SBS sleep control. The proposed energy efficiency based sleep control scheme is described in section 3. Section 4 describes the system model for performance evaluation. The results of the performance evaluation are presented in section 5 . Finally, the conclusions are given in section 6 .

\section{Related work}

\subsection{Sleep control for SBSs in HetNets}

Several SBS sleep control schemes have been proposed, for example, random and repulsive schemes are studied in [8]. Under the random scheme, SBSs are put in the sleep state with a certain probability, whereas the repulsive scheme inactivates only the SBSs within the sleeping radius of the MBS. However, these sleep controls could degrade the QoS performance of user equipments (UEs) and bring coverage holes incurred by the SBS sleeping. To solve the problems, distance-sensitive distributed repulsive sleeping strategy (DSDRSS) has been proposed in [9]. The DSDRSS scheme inactivates all the SBSs within the certain area with light traffic load. These SBSs are then merged to form a sleeping cluster. To guarantee UE coverage in that area, the DSDRSS will activate one SBS for this sleeping cluster. While DSDRSS achieves a maximum power savings of $50 \%$ by sleeping the SBSs, system throughput is also drastically decreased when compared with the no sleep control system. In [10], a sleep control scheme based on traffic load during a certain period of time has been proposed. In terms of its sleeping procedure, the activated SBSs monitor the served traffic on a regular basis. If the traffic load measured over a specified period is low, the SBS is put to sleep state to reduce power consumption. However, this technique also reduces system throughput in comparison to the no sleep control system.

\subsection{Conventional cell throughput based sleep control}

The conventional cell throughput based sleep control [11] is a simple but effective scheme applied for the SBS sleeping. The cell capacity ratio, which is a ratio of currently achieved cell throughput to maximum achievable cell throughput, is introduced as decision criteria for making SBSs sleep, in order to find the SBSs with the waste of energy. It results in SBSs with small throughput are put into the sleep state, whereas those with large cell throughput remain in the active state. Then the conventional cell throughput based sleep control scheme can reduce power consumption, and then improves energy 
efficiency.

The cell capacity ratio $R_{\mathrm{SBS}}^{i}$ for the $i$-th SBS can be obtained from the currently achieved cell throughput $S_{\mathrm{SBS}}^{i}[\mathrm{bps}]$ at the $i$-th SBS normalized by cell capacity $C_{\mathrm{SBS}}[\mathrm{bps}]$, which is formulated by:

$$
R_{\mathrm{SBS}}^{i}=\frac{S_{\mathrm{SBS}}^{i}}{C_{\mathrm{SBS}}}
$$

where $C_{\mathrm{SBS}}$ represents the maximum cell throughput achievable when the SBS fully utilizes its allocated frequency bandwidth for communication services. The $S_{\mathrm{SBS}}^{i}$ can be obtained by summing the user throughput $S_{\mathrm{UE}}^{j}$ achieved at all the UEs connecting to the $i$-th SBS, and $S_{\mathrm{SBS}}^{i}$ can be written as:

$$
\begin{aligned}
S_{\mathrm{SBS}}^{i} & =\sum_{j=1}^{N_{\mathrm{con}}^{i}} S_{\mathrm{UE}}^{j} \\
& =\sum_{j=1}^{N_{\text {con }}^{i}}\left\{B_{\mathrm{UE}}^{j} \log _{2}\left(1+\operatorname{SINR}_{\mathrm{UE}}^{j}\right)\right\}
\end{aligned}
$$

where $N_{\text {con }}^{i}$ is the number of UEs connecting to the $i$ th SBS, $B_{\mathrm{UE}}^{j}$ is the assigned frequency bandwidth to the $j$-th UE, and $S I N R_{\mathrm{UE}}^{j}$ is the received signal to interference plus noise ratio (SINR) at the $j$-th UE.

The sleep control algorithm employed in the conventional cell throughput based sleep control is shown on the inside of the dashed rectangle in the upper part of Fig. 2. The conventional cell throughput based sleep control algorithm employs a thresholdbased control for the cell capacity ratio $R_{\mathrm{SBS}}^{i}$ to determine the operation state for every SBS. The following formula is used to determine the operating state $O S^{i}$ at the $i$-th SBS:

$$
O S^{i}= \begin{cases}\text { sleep } & ; R_{\mathrm{SBS}}^{i} \leq \alpha \\ \text { active } & ; \text { otherwise }\end{cases}
$$

where $\alpha$ is a predefined threshold value for the cell capacity ratio. As shown in Eq. (3), when $R_{\mathrm{SBS}}^{i}$ is less than or equal to $\alpha$, the SBSs enter the sleep state, otherwise, the SBSs remain in the active state.

Applying this control procedure, the conventional sleep control scheme provides reduced power consumption and then improved energy efficiency, compared with no sleep control system. However, this conventional cell throughput based sleep control also causes degradation in system throughput in comparison to the no sleep control system. This is because the conventional cell throughput based sleep control excessively inactivates SBSs with a lower cell capacity rate. Then, this system throughput degradation leads to a serious problem from the perspective of efficient frequency utilization.

\subsection{Conventional adaptive SBS sleep control}

The conventional adaptive SBS sleep control [12] focuses on cell-level energy efficiency instead of system-level energy efficiency. This control proposes a sleeping technique to improve cell-level energy efficiency for active SBSs by determining and then inactivating the SBS with the minimum cell-level energy efficiency.

The conventional adaptive SBS sleep algorithm can be described as follows: after calculating the celllevel energy efficiency for all the active SBSs, the algorithm finds the SBS with minimum cell-level energy efficiency $E E_{\mathrm{SBS}}^{\min }{ }^{\circ}$. Then, the SBS with $E E_{\mathrm{SBS}}^{\mathrm{min} \text { _org }}$ temporarily changes its operating state from active to tentative sleep state. After then, the cell-level energy efficiency for all the active SBSs is recalculated, and then the updated minimum celllevel energy efficiency $E E_{\mathrm{SBS}}^{\mathrm{min} \text {-upd }}$ is derived. Finally, if the updated minimum cell-level energy efficiency is greater than the original minimum cell-level energy efficiency $\left(E E_{\mathrm{SBS}}^{\text {min_upd }}>E E_{\mathrm{SBS}}^{\mathrm{min} \_ \text {org }}\right)$, the SBS with $E E_{\mathrm{SBS}}^{\mathrm{min} \_o r g}$ changes its operating state from tentative sleep to permanent sleep state.

By employing the above control procedure, this conventional adaptive SBS sleep control achieves lower power consumption and increased energy efficiency while maintaining almost the same system throughput as the no sleep control system. However, as compared with the conventional cell throughput based sleep control [11], this conventional adaptive SBS sleep control [12] results in decreased energy efficiency, and thus it cannot provide satisfactory improvement in energy efficiency.

\section{Proposed energy efficiency based sleep control}

To solve the problems with the conventional sleep control schemes, described in the previous section, this section proposes an enhanced SBS sleep control scheme as an energy efficiency based sleep control scheme to enhance both energy efficiency and system throughput simultaneously.

Since active SBSs contribute to gain in system throughput, it is necessary to increase the number of active SBSs in order to improve system throughput. On the other hand, however, increasing the number of active SBSs results in an increased power consumption, which leads to a decrease in energy efficiency. From these considerations, energy 
efficiency must be a key element for achievable system performance from the perspectives of both the efficiencies of frequency utilization and energy consumption. Under the condition of the energy efficiency maintained without any degradation, the system can activate additional SBSs to improve system throughput. Therefore, the proposed sleep control scheme employs energy efficiency as the decision criteria for SBS operating state.

The sleep control algorithm for the proposed scheme consists of 2 phases: pre-decision phase and final-decision phase, which is shown in Fig. 2 and can be described as follows:

Pre-decision phase: The operation in this phase is applied throughout all the SBSs and is a similar operation to the conventional cell throughput based sleep control [11].

For the SBSs with cell capacity ratio $R_{\mathrm{SBS}}^{i}$ up to a predefined threshold $\alpha$, they will be temporarily considered in a "tentative sleep" state, which is a different decision from the conventional cell throughput based sleep control [11], otherwise, they remain in the active state, as shown in Eq. (4):

$$
O S^{i}= \begin{cases}\text { tentative sleep } & ; R_{\mathrm{SBS}}^{i} \leq \alpha \\ \text { active } & ; \text { otherwise }\end{cases}
$$

Final-decision phase: This operation is applied to the tentative sleep SBSs, which will be considered to be activated in this phase.

At the first step in this phase, the temporal energy efficiency $E E_{\text {sys }}^{k}[\mathrm{bps} / \mathrm{W}]$ is calculated supposing that all the tentative sleep SBSs operate in the sleep state, where $E E_{\mathrm{sys}}^{k}$ is defined as the ratio of temporal achievable system throughput $S_{\text {sys }}^{k}[\mathrm{bps}]$ to temporal power consumption $P_{\mathrm{sys}}^{k}[\mathrm{~W}]$, which can be written as:

$$
E E_{\mathrm{sys}}^{k}=\frac{s_{\mathrm{sys}}^{k}}{P_{\mathrm{sys}}^{k}}
$$

where $S_{\text {sys }}^{k}$ is defined as the summation of the achievable cell throughputs $S_{\mathrm{MBS}}^{l}$ [bps] at all the MBSs and those $S_{\text {SBS }}^{l}[\mathrm{bps}]$ at all the SBSs, which can be given by Eqs. (6) and (7), and $P_{\text {sys }}^{k}$ can be expressed as Eq. (8):

$$
\begin{gathered}
S_{\mathrm{sys}}^{k}=\sum_{l=1}^{N_{\mathrm{MBS}}} S_{\mathrm{MBS}}^{l}+\sum_{i=1}^{N_{\mathrm{SBS}}} S_{\mathrm{SBS}}^{i} \\
S_{\mathrm{MBS}}^{l}=\sum_{j=1}^{N_{\mathrm{con}}^{l}} S_{\mathrm{UE}}^{j} \\
=\sum_{j=1}^{N_{\mathrm{con}}}\left\{B_{\mathrm{UE}}^{j} \log _{2}\left(1+\operatorname{SINR}_{\mathrm{UE}}^{j}\right)\right\}
\end{gathered}
$$

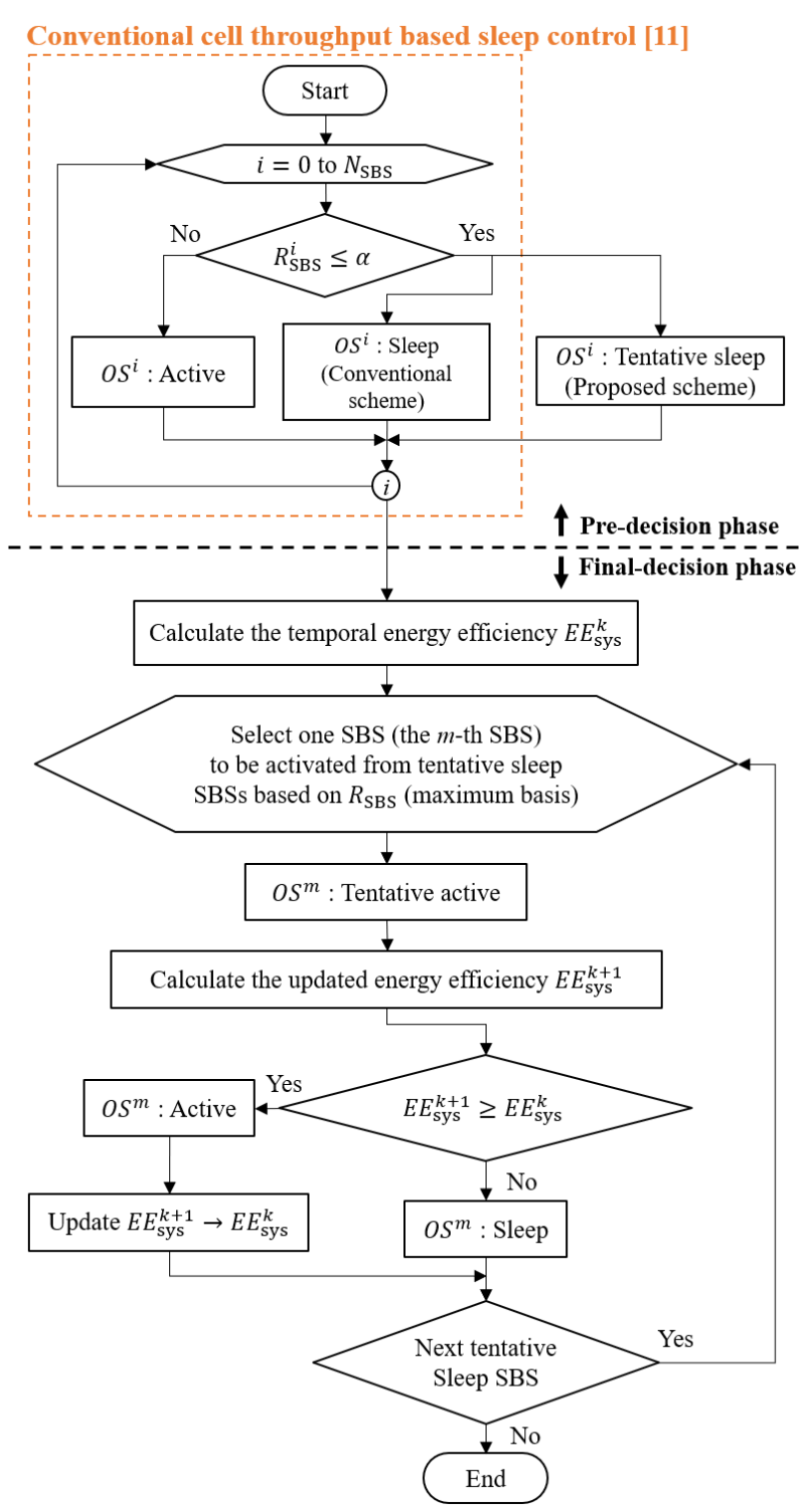

Figure. 2 The flowchart for the proposed sleep control algorithm

$$
P_{\text {sys }}^{k}=P_{\mathrm{MBS}}+P_{\text {Active }}^{k}+P_{\text {Sleep }}^{k}
$$

where $N_{\text {con }}^{l}$ is the number of UEs connected to the $l$ th MBS. Note that the detailed definition of $P_{\text {sys }}^{k}$ will be provided in Sect. 4.

Then, one SBS is chosen from the tentative sleep SBSs on the basis of the maximum cell capacity ratio $R_{\mathrm{SBS}}$, and the chosen SBS with the maximum $R_{\mathrm{SBS}}$, saying the $m$-th SBS, is changed the operating state from the tentative sleep to the active state. After then the updated energy efficiency $E E_{\text {sys }}^{k+1}$ is calculated supporting the operating state $O S^{m}$ of the chosen SBS is active.

Finally, if the updated energy efficiency $E E_{\text {sys }}^{k+1}$ is greater than or equal to the temporal energy efficiency $E E_{\text {sys }}^{k}$, which indicates no degradation in energy efficiency, the chosen SBS will eventually 
change the operating state $O S^{m}$ from the tentative sleep to the active state. Otherwise, the $O S^{m}$ changes to the sleep state, as defined in Eq. (9):

$$
O S^{m}= \begin{cases}\text { active } & ; E E_{\mathrm{sys}}^{k+1} \geq E E_{\mathrm{sys}}^{k} \\ \text { sleep } & ; \text { otherwise }\end{cases}
$$

This operation is repeated for the subsequent tentative sleep SBS until all the tentative sleep SBSs are chosen.

Through the above control procedure, the proposed sleep control scheme has the potential to simultaneously provide improved energy efficiency and almost the same system throughput as the no sleep control system, which cannot be provided by the conventional sleep control schemes.

\section{System model for performance evaluation}

This section describes the system model assumed in performance evaluations, evaluation metrics, and the schemes for performance comparison.

\subsection{System model}

The HetNet for the performance evaluation consists of two tiers of base stations: MBS tier and SBS tier. The cell layout of the MBS tier is a 2-ring structure composed of 19 cells, each with a hexagonal coverage area, as shown in Fig. 3. The MBS is located at the center of the hexagonal coverage area, and the distance between MBSs is $d_{\text {cell }}[\mathrm{m}]$. The $N_{\mathrm{SBS}}$ SBSs and $N_{\mathrm{UE}}$ UEs are distributed uniformly within a hexagonal coverage area of every MBS. The UEs are connected to either the MBS or SBS based on the maximum received signal power from them.

The network employs orthogonal frequency division multiple access (OFDMA) for radio channel access, in which the MBS or SBS allocates the same amount of the frequency bandwidth to each connecting UE by equally dividing its total frequency bandwidth $B_{\mathrm{MBS}}[\mathrm{Hz}]$ or $B_{\mathrm{SBS}}[\mathrm{Hz}]$, respectively.

The signal propagation assumes path loss due to the distance attenuation and log-normal shadow fading. Thus, the received signal power $P_{\mathrm{rx}}^{i, j}[\mathrm{~dB}]$ at the $j$-th UE for the signal from the $i$-th base station can be written as:

$$
P_{\mathrm{rx}}^{i, j}=P_{\mathrm{tx}}^{i}-\left(P L^{i, j}+\psi^{i, j}\right)
$$

where $P_{\mathrm{tx}}^{i}[\mathrm{~dB}]$ and $P L^{i, j}[\mathrm{~dB}]$ denote transmission power at the $i$-th base station (MBS or SBS), and the distance attenuation in the signal propagation path respectively. $\psi^{i, j}[\mathrm{~dB}]$ denotes its shadow fading

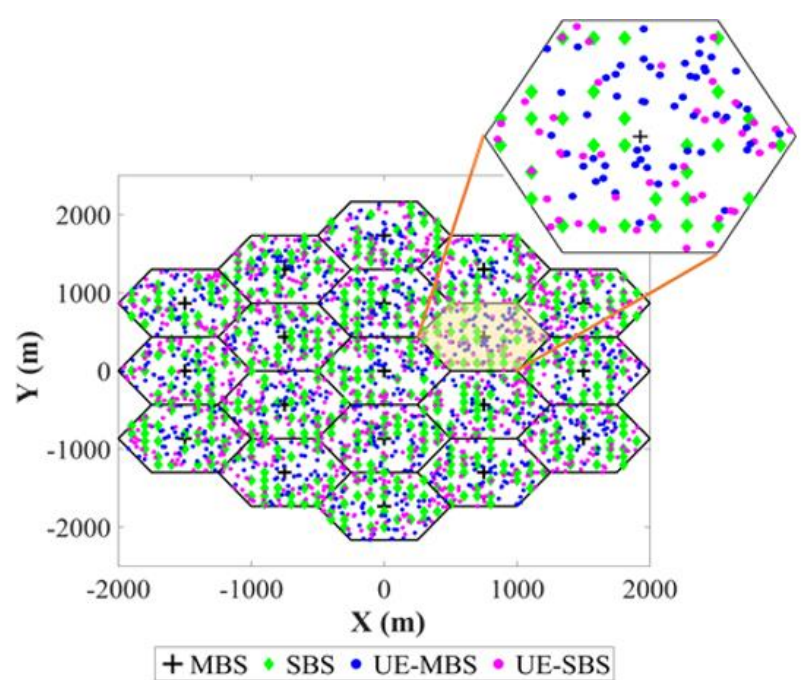

Figure. 3 BS and UE configuration under consideration

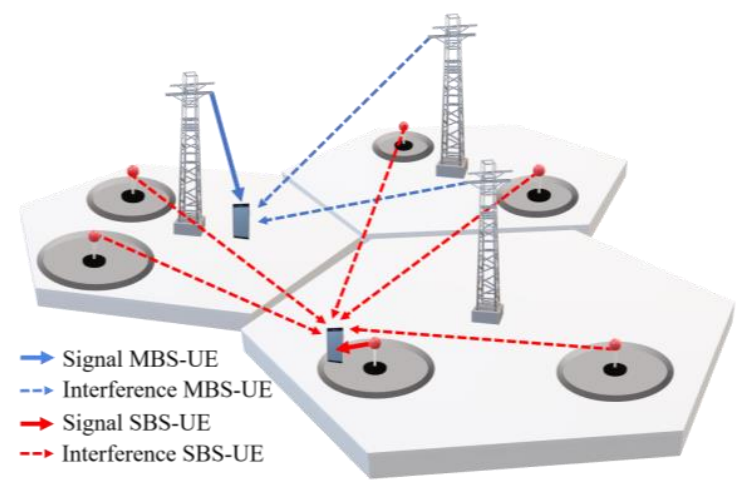

Figure. 4 Signal and interference for two-tier heterogeneous network

attenuation which follows a log-normal distribution with zero mean and standard deviation of $\sigma_{\mathrm{MBS}}$ or $\sigma_{\mathrm{SBS}}[\mathrm{dB}]$ for MBS or SBS tier. The path loss $P L^{i, j}$ is specified in the 3GPP specification TR 38.901 [13] for the carrier frequencies in $5 \mathrm{G}$ networks. For the MBS tier, the path loss $P L_{\mathrm{MBS}}^{i, j}[\mathrm{~dB}]$ can be found as Eq. (11):

$$
\begin{aligned}
P L_{\mathrm{MBS}}^{i, j}= & 13.54+39.08 \log _{10}\left(d_{3 \mathrm{D}}^{i, j}\right)+ \\
& 20 \log _{10}\left(f_{\mathrm{c}}^{\mathrm{MBS}}\right)-0.6\left(h_{\mathrm{UE}}-1.5\right)
\end{aligned}
$$

where $d_{3 \mathrm{D}}^{i, j}[\mathrm{~m}]$ denotes the 3-dimension distance between from the $i$-th MBS to the $j$-th UE, $f_{\mathrm{c}}^{\mathrm{MBS}}[\mathrm{Hz}]$ is a carrier frequency used in the MBS tier, and $h_{\mathrm{UE}}$ $[\mathrm{m}]$ is an antenna height for UEs. The path loss $P L_{\mathrm{SBS}}^{i, j}$ $[\mathrm{dB}]$ for the SBS tier is defined in Eq. (12), where $f_{\mathrm{c}}^{\mathrm{SBS}}[\mathrm{Hz}]$ defines a carrier frequency for the SBS tier: 


$$
\begin{aligned}
P L_{\mathrm{SBS}}^{i, j}= & 22.4+35.3 \log _{10}\left(d_{3 \mathrm{D}}^{i, j}\right)+ \\
& 21.3 \log _{10}\left(f_{\mathrm{c}}^{\mathrm{SBS}}\right)-0.3\left(h_{\mathrm{UE}}-1.5\right)
\end{aligned}
$$

The network employs the different carrier frequency bands $f_{\mathrm{c}}^{\mathrm{MBS}}$ and $f_{\mathrm{c}}^{\mathrm{SBS}}$ between the MBS and SBS tiers. The network does not employ frequency reuse technique for both the MBS and SBS tiers. Therefore, the co-channel interference at the UE is caused by the arriving signals from all the BSs except its own connected BS in the same tier as shown in Fig. 4.

As for the SINR $\operatorname{SINR}_{\mathrm{MBS}}^{j}$ at the $j$-th $\mathrm{UE}$ connecting to the $i$-th MBS and that $\operatorname{SINR}_{\mathrm{SBS}}^{j}$ at the $j$-th UE connecting to the $i$-th SBS can be expressed by [14]:

$$
\begin{aligned}
& \operatorname{SINR}_{\mathrm{MBS}}^{j}=\frac{P_{\mathrm{rX}}^{i, j}}{I_{\mathrm{MBS}}^{j}+N_{0} B_{\mathrm{MBS}}^{j}} \\
& \operatorname{SIN} R_{\mathrm{SBS}}^{j}=\frac{P_{\mathrm{rX}}^{i, j}}{I_{\mathrm{SBS}}^{j}+N_{0} B_{\mathrm{SBS}}^{j}}
\end{aligned}
$$

where $N_{0}[\mathrm{dBm} / \mathrm{Hz}]$ denotes noise power density, $B_{\mathrm{MBS}}^{j}$ and $B_{\mathrm{SBS}}^{j}[\mathrm{~Hz}]$ are the frequency bandwidth allocated to the $j$-th UE by the connected $i$-th MBS and SBS, respectively. The $I_{\mathrm{MBS}}^{j}$ and $I_{\mathrm{SBS}}^{j}$ denote the total co-channel interference at the $j$-th UE from other BSs in the same BS tier, except its own connected BS, which are defined by:

$$
\begin{aligned}
& I_{\mathrm{MBS}}^{j}=\sum_{i \in \emptyset_{\mathrm{MBS}}^{j}} P_{\mathrm{rX}}^{i, j} \\
& I_{\mathrm{SBS}}^{j}=\sum_{i \in \emptyset_{\mathrm{SBS}}^{j}} P_{\mathrm{rX}}^{i, j}
\end{aligned}
$$

where $\emptyset_{\mathrm{MBS}}^{j}$ and $\emptyset_{\mathrm{SBS}}^{j}$ represent the set of BSs interfering to the $j$-th UE.

Only the downlink communication from BSs to UEs is assumed and its data traffic employs a full buffer model. Therefore, the UE throughput $S_{\mathrm{UE}}^{j}$ [bps] at the $j$-th UE can be derived by using Shannon's channel capacity theory in Eq. (17) [15]:

$$
S_{\mathrm{UE}}^{j}=B_{\mathrm{UE}}^{j} \log _{2}\left(1+\operatorname{SIN} R_{\mathrm{UE}}^{j}\right)
$$

where $B_{\mathrm{UE}}^{j}$ is either of $B_{\mathrm{MBS}}^{j}$ or $B_{\mathrm{SBS}}^{j}$ depending on the BS tier connected by the $j$-th UE. $\operatorname{SINR}_{\mathrm{UE}}^{j}$ is also either of $\operatorname{SINR}_{\mathrm{MBS}}^{j}$ or $\operatorname{SINR}_{\mathrm{SBS}}^{j}$.

For the power consumption at each BS, this paper focuses on the power consumption at the transceiver on the BSs, which is typically consisted of a power amplifier (PA) module, a radio frequency signal (RF) module, a baseband processing (BB) module, a DCDC power supply (DCDC), an active cooling system (CO), and an AC-DC (main supply) unit (ACDC) [16-17]. For the power consumption at the SBS, we assume different states for SBS operations: active, idle, and sleep. The active state is defined as the state in which the SBS is fully operational, including the operation of the PA and RF modules with maximum power due to the provision of communication services to connected UE. In the idle state, the SBS is partially operational, which means the SBS does not operate especially the PA and RF modules due to no requirement of communication services to UEs under the case with no connected UEs, and consumes lower power than the active state. In the sleep state, the SBS does not operate major operating parts, however, to ensure that it could be activated, it should not be entirely powered off, and then may continue to consume some power.

Each operating state is assumed to consume the different power: $P_{\text {Active }}, P_{\text {Idle }}$, and $P_{\text {Sleep }}[\mathrm{W}]$ for active, idle, and sleep state, respectively. For the power consumption at the MBS, all the MBSs always active, and then their power consumption $P_{\mathrm{MBS}}[\mathrm{W}]$ is assumed to be constant.

\subsection{Evaluation metrics}

In the performance evaluation, system throughput $S_{\text {sys }}$, system power consumption $P_{\text {sys }}$, and energy efficiency $E E_{\text {sys }}$ are evaluated by computer simulation.

The system throughput $S_{\text {sys }}[\mathrm{bps}]$ is defined as the summation of the cell throughputs for all the MBSs and SBSs with their final operation state in the HetNets, which is calculated by applying Eqs. (2), (6), and (7).

The system power consumption $P_{\text {sys }}[\mathrm{W}]$ is defined as the total power consumed at all the base stations. Thus, $P_{\text {sys }}$ can be expressed by:

$$
\begin{aligned}
P_{\text {sys }}= & \left(P_{\text {MBS }} \cdot N_{\text {MBS }}\right)+\left(P_{\text {Active }} \cdot N_{\text {Active }}\right)+ \\
& \left(P_{\text {Idle }} \cdot N_{\text {Idle }}\right)+\left(P_{\text {Sleep }} \cdot N_{\text {Sleep }}\right)
\end{aligned}
$$

where $N_{\text {Active }}, N_{\text {Idle }}$, and $N_{\text {Sleep }}$ are the number of SBSs under the final operating states of active, idle, and sleep, respectively.

The energy efficiency $E E_{\text {sys }}[\mathrm{bps} / \mathrm{W}]$ is defined as an achieved system throughput $S_{\text {sys }}$ normalized by the power consumption $P_{\text {sys }}$, which is calculated by applying Eq. (5) [18]. 


\subsection{Schemes under comparison}

For the performance evaluation, this paper compares the performances for four different schemes.

- No sleep control system: All the SBSs remain in the active state, except for the SBSs with no UEs connected, which are under an idle state.

- The conventional cell throughput based sleep control [11], which employs the cell capacity ratio as a decision criteria, described in sect. 2.2.

- The conventional adaptive SBS sleep control [12], which inactivates the SBS with the minimum cell-level energy efficiency, explained in sect. 2.3.

- The proposed sleep control, described in sect. 3 .

\section{Performance evaluation}

This section presents the computer simulation results for four different schemes. All simulations were conducted using MATLAB software and included monte carlo simulations for $10^{5}$ trials to ensure statistically significant results. The system throughput $S_{\text {sys }}$, system power consumption $P_{\text {sys }}$, and energy efficiency $E E_{\text {sys }}$ are shown while comparing between four schemes. The performances for the sleeping operation rate $S O R_{\mathrm{SBS}}$ also shown in this section.

\subsection{Simulation parameters and their settings}

We use the parameter settings for the simulation parameters listed in Table 1. The total number of SBSs and UEs per MBS coverage is defined between 5 to 50 and 50 to 300 , respectively. The frequency

bandwidth is $20 \mathrm{MHz}$ for both MBSs and SBSs. The carrier frequencies for MBS and SBS tier are $0.8 \mathrm{GHz}$ and $3.7 \mathrm{GHz}$, respectively [19]. The transmission power at MBSs and SBSs is $46 \mathrm{dBm}$ and $30 \mathrm{dBm}$, respectively. The threshold $\alpha$ for cell capacity ratio is set as a constant value of 0.25 , which is an optimum value for the energy efficiency in the conventional cell throughput based sleep control scheme and proposed sleep control scheme.

\subsection{System throughput performance}

Fig. 5 (a) shows the system throughput $S_{\text {sys }}$ for varying the number of SBSs per MBS for the number of UEs $N_{\mathrm{UE}}=100$. The proposed sleep control scheme improves system throughput $S_{\text {sys }}$ over the conventional cell throughput based sleep control
Table 1. Simulation parameter setting [11, 13]

\begin{tabular}{lc|rl}
\hline & Symbol & \multicolumn{2}{|c}{ Value } \\
\hline Distance between & $d_{\text {cell }}$ & $500 \sqrt{3}$ & {$[\mathrm{~m}]$} \\
MBSs & $N_{\mathrm{MBS}}$ & 19 & \\
No. of MBS & $N_{\mathrm{SBS}}$ & $5-50$ & \\
No. of SBS [/MBS] & $N_{\mathrm{UE}}$ & $50-300$ & \\
No. of UE [/MBS] & $B_{\mathrm{MBS}}, B_{\mathrm{SBS}}$ & 20 & {$[\mathrm{MHz}]$} \\
Bandwidth & $f_{\mathrm{c}}^{\mathrm{MBS}}, f_{\mathrm{c}}^{\mathrm{SBS}}$ & $0.8,3.7$ & {$[\mathrm{GHz}]$} \\
Carrier frequency at & & & \\
MBS, SBS & $P_{\mathrm{tx}}^{\mathrm{MBS}}, P_{\mathrm{tx}}^{\mathrm{SBS}}$ & 46,30 & {$[\mathrm{dBm}]$} \\
TX power at MBS, & & & \\
SBS & $\sigma_{\mathrm{MBS}}, \sigma_{\mathrm{SBS}}$ & $6,7.82$ & {$[\mathrm{~dB}]$} \\
Shadow fading std & & -174 & {$[\mathrm{dBm} /$} \\
for MBS, SBS & $N_{0}$ & & $\mathrm{~Hz}]$ \\
Noise power density & $\alpha$ & 0.25 & \\
Cell capacity & $P_{\mathrm{MBS}}$ & 150 & \\
threshold & $P_{\text {Active }}$ & 30 & {$[\mathrm{~W}]$} \\
\hline $\begin{array}{l}\text { Power consumption } \\
\text { at MBS, Active, Idle, }\end{array}$ & $P_{\mathrm{Idle}}$ & 25 & \\
Sleep & $P_{\text {Sleep }}$ & 15 & \\
\hline
\end{tabular}

scheme [11] and achieves almost the same as no sleep control system and the conventional adaptive sleep control scheme [12]. This is because the proposed one can activate more SBSs, compared with the cell throughput based scheme [11], and this activation contributes to an improvement in the system throughput. As can be observed in Fig. 5 (a), the proposed scheme achieves more improvement in the system throughput over the cell throughput based sleep control scheme, especially for the case with the larger number of SBSs $N_{\mathrm{SBS}}$ from 30 to 50 . For example, at $N_{\mathrm{SBS}}=50$, the proposed scheme achieves only $0.6 \%$ degradation from the no sleep control system, whereas the conventional cell throughput based sleep control scheme [11] shows $3.1 \%$ degradation.

The system throughput $S_{\text {sys }}$ for varying the number of UEs per MBS is also shown in Fig. 5 (b) for the case of $N_{\mathrm{SBS}}=30$. The proposed sleep control scheme also provides almost the same system throughput as no sleep control system and the conventional adaptive sleep control scheme [12] for any number of UEs. The reason for this improvement is the same as that in Fig. 5 (a). Furthermore, the proposed scheme largely improves system throughput for the case with the smaller number of UEs $N_{\mathrm{UE}}$ from 50 to 150 . This improvement under such the case, as well as the case with the larger number of SBSs in Fig. 5 (a), is caused by the fact that the network has the large number of candidate SBSs to be activated under such the cases, as can be 


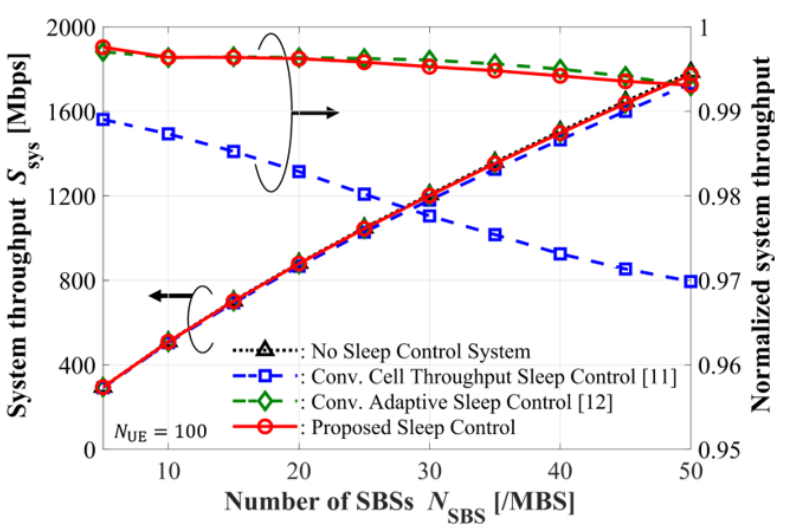

(a)

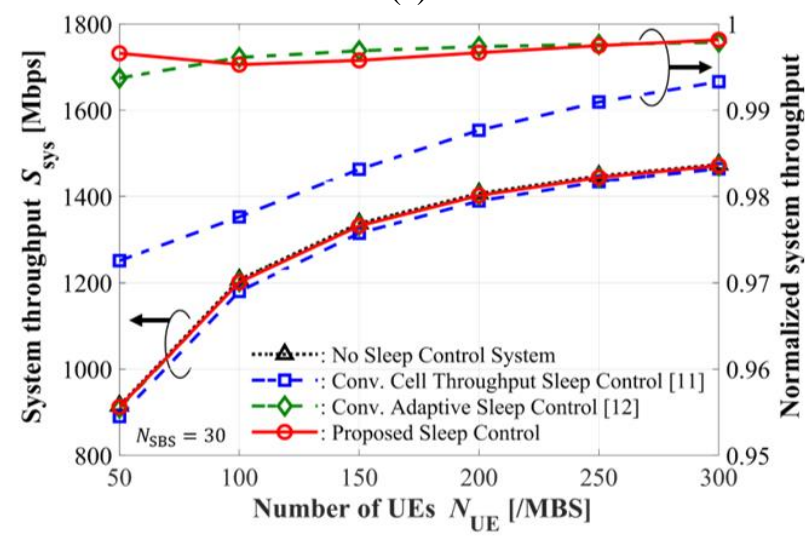

(b)

Figure. 5 The system throughput $S_{\text {sys }}$ for different numbers of (a) SBSs $N_{\mathrm{SBS}}$ and (b) UEs $N_{\mathrm{UE}}$

found in Fig. 6, which shows the sleeping operation rate under the same condition as Fig. 5. We can find in Fig. 6 that the sleeping operation rate is relatively high under such the cases, and then the network has more SBSs to be activated in the final decision phase by the proposed scheme, resulting in a larger improvement in the system throughput.

In addition, as illustrated in Fig. 5, the proposed scheme shows the constant normalized system throughput, which is defined as a ratio of the achievable system throughput to that for the no sleep control system, for any numbers of SBSs and UEs. In contrast to the proposed scheme, the normalized system throughput for the conventional cell throughput based sleep control scheme [11] depends on the number of SBSs and UEs, and then, the conventional scheme [11] cannot provide the constant normalized system throughput for all the system conditions.

\subsection{Power consumption performance}

Fig. 7 shows the system power consumption $P_{\text {sys }}$ under the same conditions as Fig. 5. It is observed that the power consumption $P_{\text {sys }}$ for all the sleep control

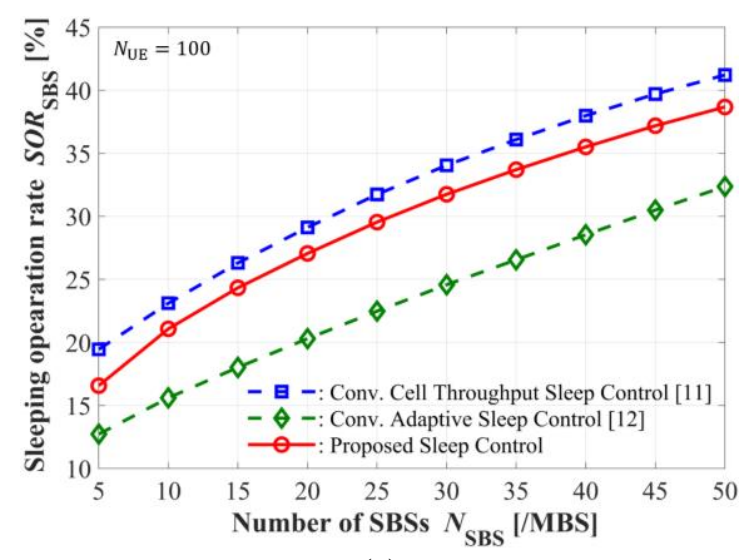

(a)

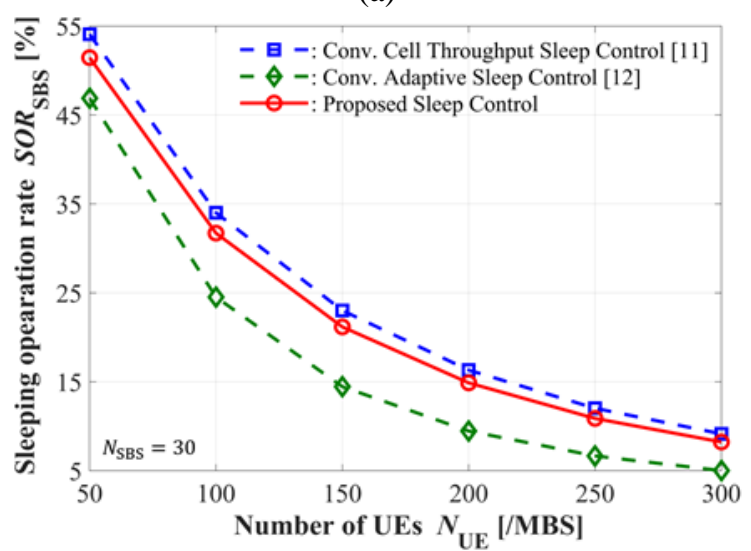

(b)

Figure. 6 The sleeping operation rate $S O R_{\mathrm{SBS}}$ for different numbers of (a) SBSs $N_{\mathrm{SBS}}$ and (b) UEs $N_{\mathrm{UE}}$

schemes are less than that for the no sleep control system. However, the conventional adaptive sleep control scheme [12] consumes larger power than the proposed scheme and conventional cell throughput based sleep control scheme [11]. The proposed scheme achieves the smallest $P_{\text {sys }}$ performance, however it shows slightly larger $P_{\text {sys }}$ than the conventional cell throughput based sleep control scheme [11]. This is due to the fact that the proposed scheme activates additional SBSs from the tentative sleep SBSs in its final-decision phase. As a result, the proposed sleep control scheme activates a larger number of SBSs, leading to slightly larger power consumption.

Moreover, the proposed scheme shows slightly larger degradation in the power consumption for the cases with larger improvement in the system throughput, that is the cases with larger $N_{\mathrm{SBS}}$ or smaller $N_{\mathrm{UE}}$. This means the proposed scheme provides throughput improvement with a cost of power consumption. 


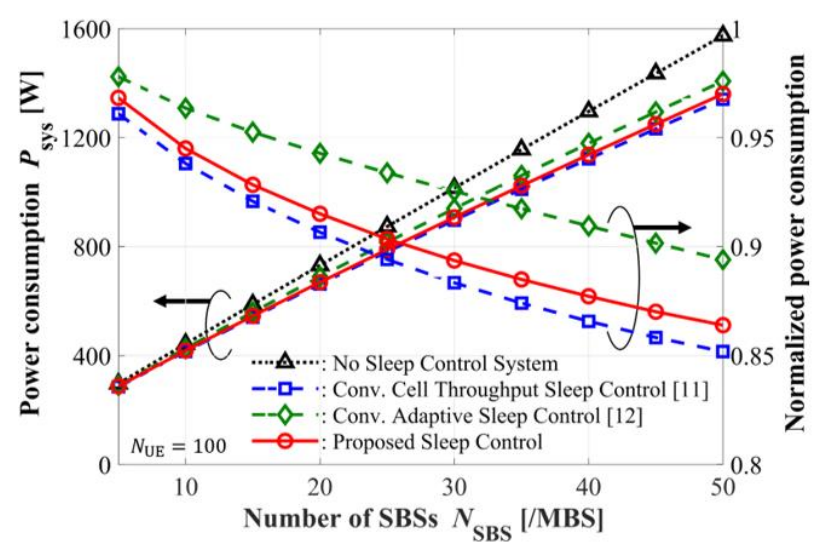

(a)

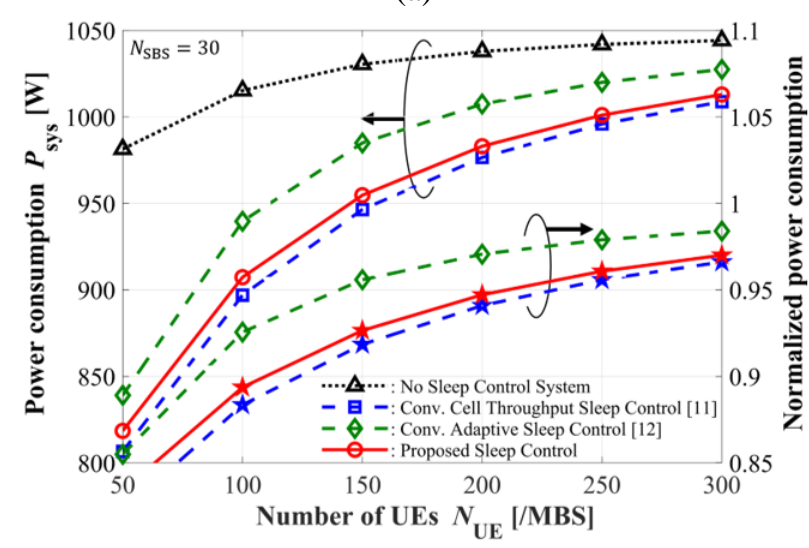

(b)

Figure. 7 The power consumption $P_{\text {sys }}$ for different numbers of (a) SBSs $N_{\mathrm{SBS}}$ and (b) UEs $N_{\mathrm{UE}}$

\subsection{Energy efficiency performance}

Fig. 8 shows the energy efficiency $E E_{\text {sys }}$ under the same conditions as Fig. 5. The proposed scheme can achieve superior $E E_{\text {sys }}$ performance to both the conventional sleep control schemes, particularly compared with the conventional adaptive sleep control scheme [12], which provides almost the same system throughput. This is because the proposed scheme employs energy efficiency as the criteria for the decision of the SBS operating state in its finaldecision phase prior to activating additional SBSs for the purpose of improving system throughput. The performance gain in the energy efficiency by the proposed scheme gets larger for the cases with larger improvement in the system throughput. This fact shows the gain in the system throughput surpasses the cost in the energy consumption in the proposed scheme.

Table 2 compares the energy efficiency $E E_{\text {sys }}$ achieved by different schemes for different numbers of SBSs $N_{\text {SBS. }}$. We can find from Table 2 that the proposed sleep control scheme can provide the highest $E E_{\text {sys }}$ of for example 1.304 [Mbps/W] at

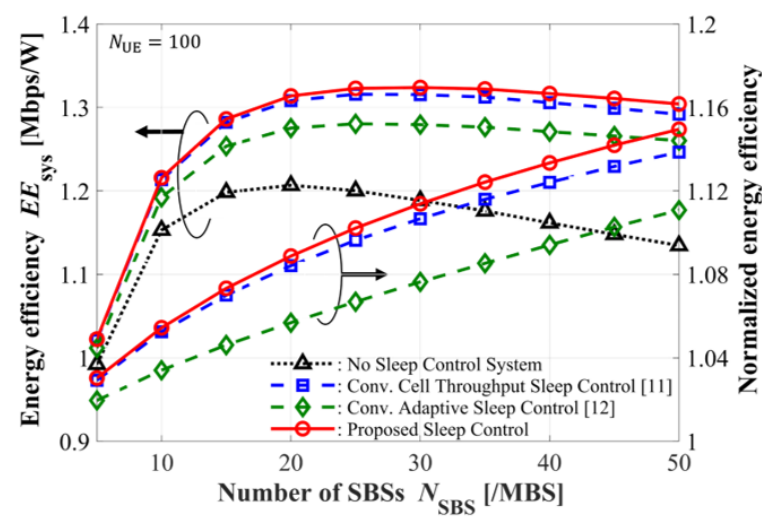

(a)

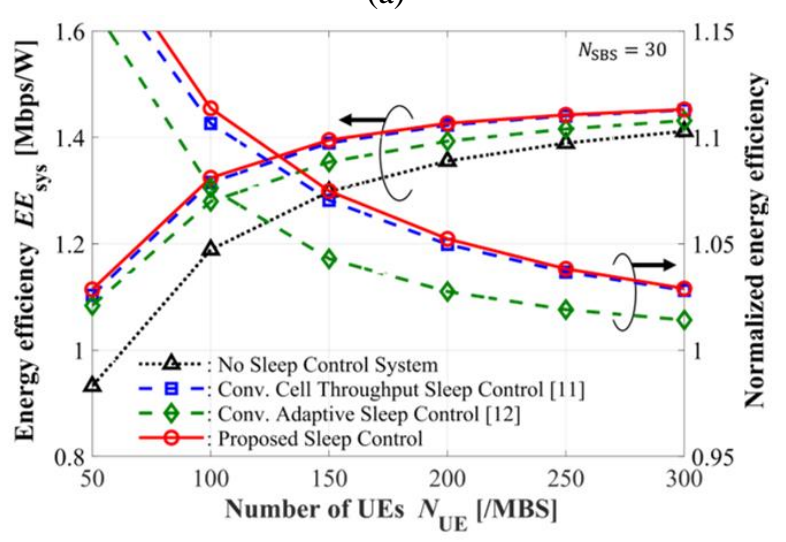

(b)

Figure. 8 The energy efficiency $E E_{\text {sys }}$ for different numbers of (a) SBSs $N_{\mathrm{SBS}}$ and (b) UEs $N_{\mathrm{UE}}$

Table 2. Comparison of energy efficiency $E E_{\text {sys }}$ for different numbers of SBSs $N_{\mathrm{SBS}}$ and $N_{\mathrm{UE}}=100$

\begin{tabular}{|l|c|c|c|}
\hline \multirow{2}{*}{\multicolumn{1}{|c}{ Sleep Control Schemes }} & \multicolumn{3}{|c|}{$\boldsymbol{N}_{\text {SBS }}$} \\
\cline { 2 - 4 } & $\mathbf{3 0}$ & $\mathbf{4 0}$ & $\mathbf{5 0}$ \\
\hline No sleep control system & 1.189 & 1.162 & 1.135 \\
\hline $\begin{array}{l}\text { Conv. cell throughput sleep } \\
\text { control [11] }\end{array}$ & 1.315 & 1.306 & 1.292 \\
\hline Conv. adaptive sleep control [12] & 1.279 & 1.271 & 1.260 \\
\hline Proposed sleep control & 1.324 & 1.317 & 1.304 \\
\hline
\end{tabular}

$N_{\text {SBS }}=50$, which is a $14.89 \%$ improvement in comparison to the no sleep control system, whereas the conventional cell throughput based scheme [11] and the conventional adaptive sleep control scheme [12] improve performance by only $13.83 \%$ and $11.01 \%$, respectively.

\section{Conclusions}

This paper discussed the sleep control applied for the SBSs in 5G HetNets. Aiming at improving energy efficiency and system throughput simultaneously, this paper proposed the enhanced sleep control algorithm which employs energy efficiency as a decision criterion for switching the SBS state into active or sleep state. The proposed sleep control 
introduces a 2-phase control procedure and its second phase activates additional SBSs from the sleep SBSs to improve the energy efficiency while retaining the system throughput.

We evaluated the system throughput, power consumption, and energy efficiency for the proposed scheme and compared with the conventional schemes. The evaluation results through computer simulation demonstrate that, the proposed sleep control scheme achieves superior energy efficiency and system throughput simultaneously, indicating that it can improve energy efficiency while maintaining almost the same system throughput as no sleep control system, which cannot be provided by the conventional sleep control schemes. Moreover, the proposed scheme improves energy efficiency by $14.89 \%$ while maintaining almost the same system throughput of over $99 \%$ when compared with the no sleep control system. Consequently, the proposed scheme can contribute to providing high capacity green HetNets for 5G NR cellular networks.

In future work, the proposed sleep control scheme could be applied to the HetNets with dynamic behavior, such as UE mobility and traffic fluctuation, by enhancing our proposed sleep control so as to handle the dynamic behavior in HetNets.

\section{Conflicts of interest}

The authors declare no conflict of interest.

\section{Author contributions}

Conceptualization, Arif Dataesatu and Kazuo Mori; methodology, Arif Dataesatu and Kazuo Mori; software, Arif Dataesatu; validation, Kazuo Mori; formal analysis, Arif Dataesatu and Kazuo Mori; investigation, Arif Dataesatu and Kazuo Mori; resources, Arif Dataesatu; data curation, Arif Dataesatu; writing — original draft preparation, Arif Dataesatu and Kazuo Mori; writing - review and editing, Pisit Boonsrimuang, Kosuke Sanada and Hiroyuki Hatano; supervision, Kazuo Mori.

\section{References}

[1] 3GPP TR 38.913 V14.3.0, "Study on Scenarios and Requirements for Next Generation Access Technologies (Release 14)", 2017.

[2] E. Hossain and M. Hasan, "5G Cellular: Key Enabling Technologies and Research Challenges", IEEE Instrumentation and Measurement Magazine, Vol. 18, No. 3, pp. 1121, 2015.

[3] A. Radwan, M. F. Domingues, and J. Rodriguez, "Mobile Caching Enabled Small-Cells for
Delay-Tolerant e-Health Apps", In: Proc. of IEEE International Conference on Communications Workshops (ICC Workshops), pp. 103-108, 2017.

[4] S. Chou, T. Chiu, Y. Yu, and A. Pang, "Mobile Small Cell Deployment for Next Generation Cellular Networks", In: Proc. of IEEE Global Communications Conference (GLOBECOM), pp. 4852-4857, 2014.

[5] M. Agiwal, A. Roy, and N. Saxena, "Next Generation 5G Wireless Networks: A Comprehensive Survey", IEEE Communications Surveys \& Tutorials, Vol. 18, No. 3, pp. 1617-1655, 2016.

[6] J. Wu, Y. Zhang, M. Zukerman, and E. K. Yung, "Energy-Efficient Base-Stations Sleep-Mode Techniques in Green Cellular Networks: A Survey", IEEE Communications Surveys \& Tutorials, Vol. 17, No. 2, pp. 803-826, 2015.

[7] F. Salahdine, J. Opadere, Q. Liu, T. Han, N. Zhang, and $\mathrm{S}$. $\mathrm{Wu}$, "A survey on sleep mode techniques for ultra-dense networks in $5 \mathrm{G}$ and beyond", Computer Networks, Vol. 201, 2021.

[8] S. Zhang, J. Gong, and S. Zhou, "How Many Small Cells Can be Turned Off via Vertical Offloading Under a Separation Architecture?", IEEE Transactions on Wireless Communications, Vol. 14, No. 10, pp. 5440 5453, 2015.

[9] Y. He, L. Tang, and Z. Zhou, "A DistanceSensitive Distributed Repulsive Sleeping Strategy for Densely-Deployed Small Cells in Green Cities", In: Proc. of IEEE International Conference on Communications (ICC), pp. 211216, 2018.

[10] L. Falconetti, L. Hevizi, and I. Godor, "Sleep Mode Control for Low Power Nodes in Heterogeneous Networks", In: Proc. of The Tenth International Symposium on Wireless Communication Systems (ISWCS), pp. 1-5, 2013.

[11] P. Phaiwitthayaphorn, K. Mori, H. Kobayashi, and P. Boonsrimuang, "Cell Throughput based Sleep Control Scheme for Heterogeneous Cellular Networks", ECTI Transactions on Computer and Information Technology, Vol. 12, No. 1, pp. 26-33, 2018.

[12] S. Habibi, V. Solouk, and H. Kalbkhani, "Adaptive Energy-Efficient Small Cell Sleeping and Zooming in Heterogeneous Cellular Networks", Telecommunication Systems, Vol. 77, No. 1, pp. 23-45, 2021.

[13] 3GPP TR 38.901 V16.1.0, "Study on Channel Model for Frequencies from 0.5 to $100 \mathrm{GHz}$ (Release 16)", 2019. 
[14] K. R. Krishnan and H. Luss, "Power Selection for Maximizing SINR in Femtocells for Specified SINR in Macrocell", In: Proc. of IEEE Wireless Communications and Networking Conference (WCNC), pp. 563-568, 2011.

[15] D. Tse and P. Viswanath, Fundamentals of Wireless Communication, Cambridge University Press, 2005.

[16] V. Giannini, C. Desset, and I. Godor, "How Much Energy is Needed to Run a Wireless Network?", IEEE Wireless Communications, Vol. 18, No. 5, pp. 40-49, 2011.

[17] A. Israr, Q. Yang, W. Li, and A. Y. Zomaya, "Renewable Energy Powered Sustainable 5G Network Infrastructure: Opportunities, Challenges and Perspectives", Journal of Network and Computer Applications, Vol. 175, 2021.

[18] Y. Chen, S. Zhang, and S. Xu, "Characterizing Energy Efficiency and Deployment Efficiency Relations for Green Architecture Design", In: Proc. of IEEE International Conference on Communications Workshops (ICC Workshops), pp. 1-5, 2010.

[19] Y. Sagae, S. Sawamukai, Y. Ohwatari, K. Kiyoshima, K. Kanbara, and J. Takahashi, "5G Network", NTT DOCOMO Technical Journal, Vol. 22, No. 2, pp. 23-39, 2020. 